\title{
Physics Education Using 3D Antenna Simulations
}

\section{Georgi Golemshinski ${ }^{1}$}

\author{
${ }^{1}$ Researcher in the field of e-learning, Geophysics and Chemistry, Bulgaria
}

\begin{abstract}
E-learning physics using visualizations of certain physical characteristics of the studied phenomenon are described. Teaching electromagnetism and radio-electronics, the fundamentals of radio-wave propagation and antenna construction may be realized effectively using $3 D$ visualization, either through static or dynamic $3 D$ graphics. The author tries to establish an e-learning route for implementing modern learning methods based on information technologies in radio physics and electromagnetism courses in universities. The current paper discloses the benefits of implementing free access $3 D$ software for e-learning purposes through analyzing helix antenna in axial mode.
\end{abstract}

Keywords: E-learning, 3D antenna simulations in teaching physics

\section{Introduction}

The current article shows an e-learning method of teaching physics and electrodynamical phenomena to students in universities. The author has studied the inevitable advance of e-learning methods in different areas of education during the last few years [1-2]. This material focuses on the implementation of $3 \mathrm{D}$ visualizations and simulations in the field of physics education. Such recent developments are presented in a number of publications dealing with elearning physics using 3D online simulations [3-4].

What is most important about $3 \mathrm{D}$ visualization is the ability to perceive otherwise invisible characteristics of the studied process or to observe its visible characteristics in an environment being hard or impossible to establish in the laboratory.

This visualization is three dimensional either as an image, i.e. statically, or as a rotating $3 \mathrm{D}$ scene.

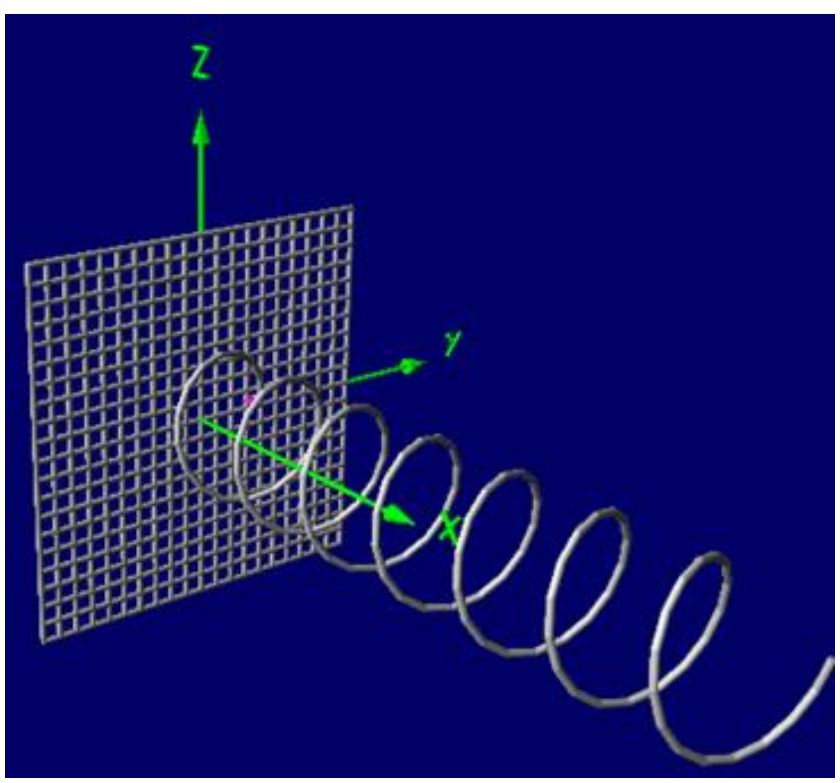

Figure 1: Helix antenna structure in 3D.

\section{The Helical Antenna in Axial Mode}

In the current material the helical antenna used in axial mode is presented as a setup to show how implementing free access 3D software for antenna analysis and visualization can be beneficial to students attending physics and related to physics and radio electronics courses in universities (see fig. 1 and fig. 2).

This antenna has many features that make it much suitable for e-learning radio-electronics and antenna theory. All these features are readily demonstrated using $3 \mathrm{D}$ visualization as described in the following paragraphs.

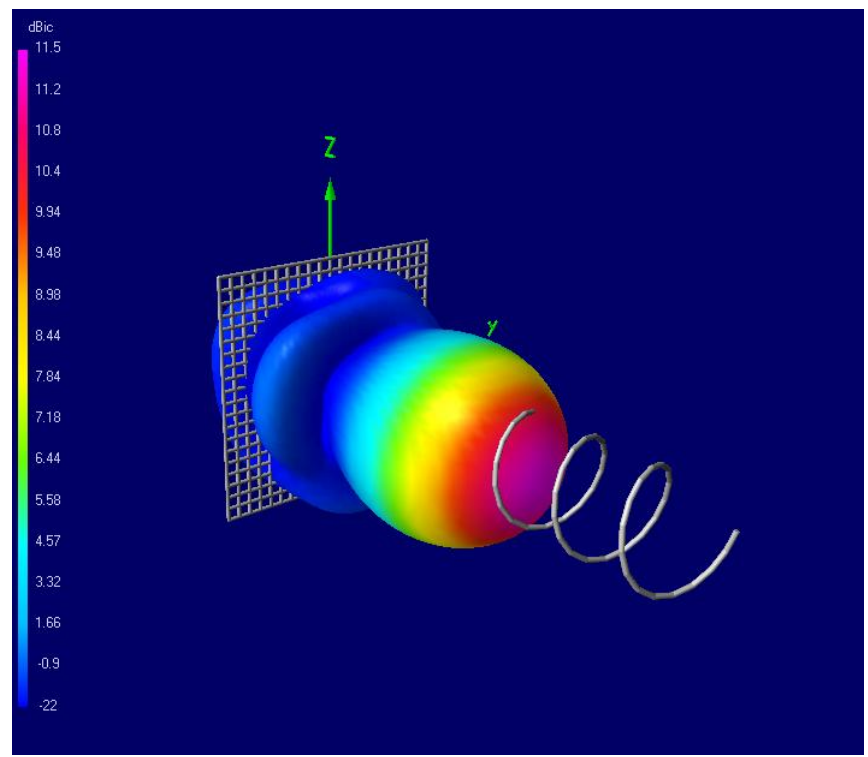

Figure 2: Helix antenna total gain in 3D

\section{Antenna Gain and 3D visualization}

Another pronounced benefit of the helix antenna is its high gain in the direction of the helix axis (see fig. 2 and fig. 7). The gain depends on the number of turns, but antennas implementing more than 12 turns are rarely used due to increased losses. The presented model has 7 turns and exhibits total gain of $11.5 \mathrm{dBic}$. The $3 \mathrm{D}$ visualization of the gain pattern with color palette gives good comprehension of the pattern itself and of the maximum gain. The observer may also identify the backwards gain and hence the front to back ratio of the antenna. 


\section{International Journal of Science and Research (IJSR) \\ ISSN (Online): 2319-7064}

Index Copernicus Value (2013): 6.14 | Impact Factor (2014): 5.611

The drawback of the helical antenna high gain is its relatively large size. That's why this antenna is used at VHF and higher frequencies only.

\section{Antenna Polarization and $3 D$ visualization}

The helical antenna in axial mode is circularly polarized. Circular polarization is beneficial where the mutual relative orientation of the receiving and transmitting antennas is not kept constant. This situation happens in aerospace communications and with the advent of modern drones has become very important issue. Another reason for why circular polarization may be used is the reflection of the radio signal from obstacles. The circular polarization will cancel all odd reflections in the receiving antenna, i.e. all odd reflections will have opposite circular polarization. The first reflection being of largest magnitude will be cancelled. The helical antenna can be right- or left-hand polarized according to how the helix is twisted. The current article demonstrates right-hand polarized antenna (see fig. 3 and fig. 4).

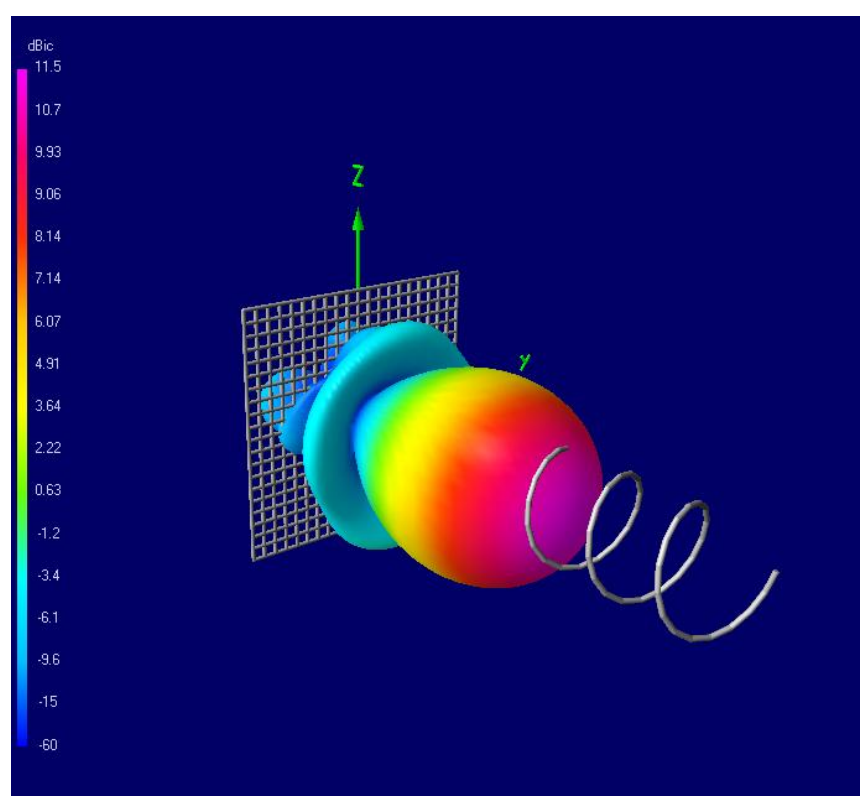

Figure 3: Helix antenna right-hand circular polarization (RHCP) gain in $3 \mathrm{D}$.

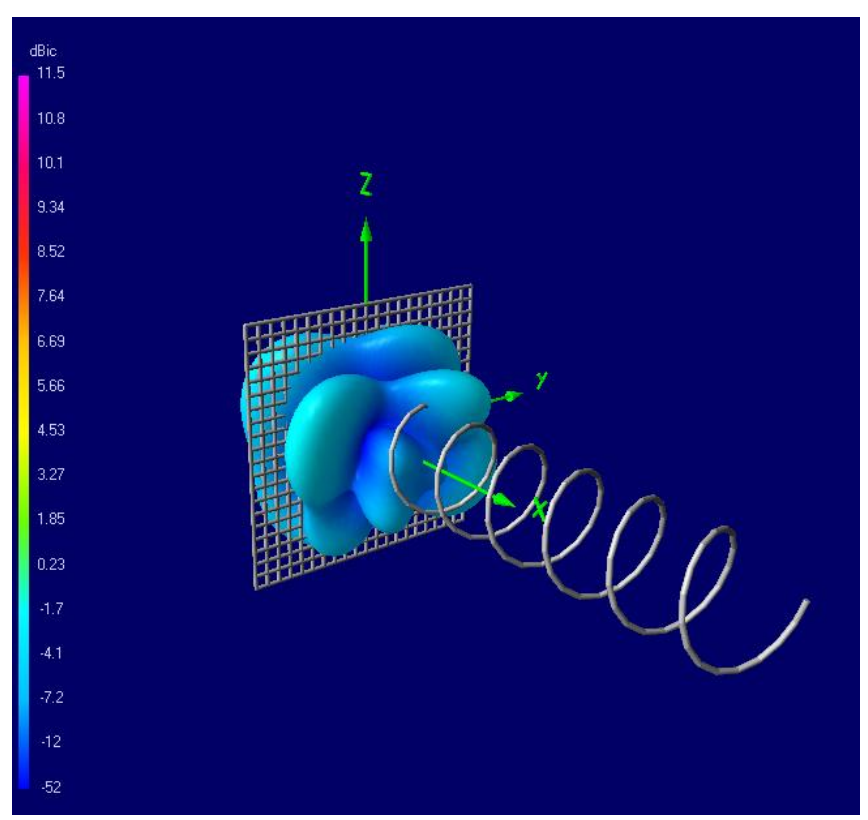

Figure 4: Helix antenna left-hand circular polarization LHCP gain in $3 \mathrm{D}$.

Although polarized, the helical antenna may receive signals with any type of linear polarization, i.e. horizontal, vertical or diagonal polarization, but with diminished gain with 3 dB. When receiving circularly polarized signals the polarization, either right-handed or left-handed, must be established in both the receiving and the transmitting antennas.

The circularly polarized gain of the antenna may be observed using the 3D visualization. The student may easily identify the right-hand gain as being large and the left-hand gain being extremely small. Although small, the reverse polarization is still present and has different gain pattern than the main polarization gain (see fig. 3, fig. 4 and fig. 5).

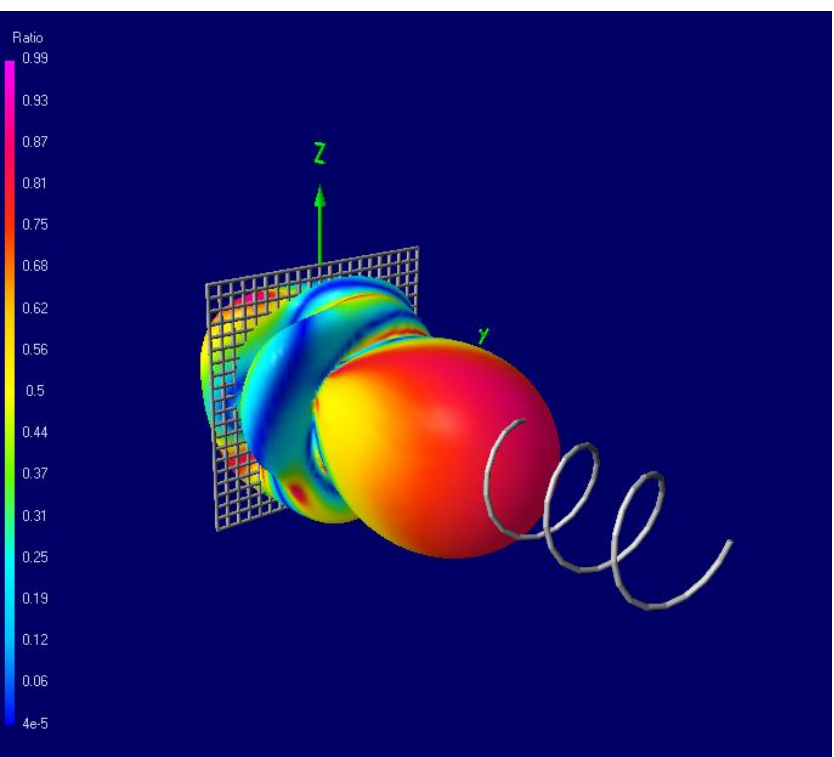

Figure 5: Helix antenna axial ratio in 3D.

The axial ratio of the gain is the characteristic that demonstrates the imperfectness of the circular polarization (see fig. 4). The circle that describes the circular polarization is not a perfect one but rather is an ellipse. The axial ratio is 


\section{International Journal of Science and Research (IJSR) \\ ISSN (Online): 2319-7064 \\ Index Copernicus Value (2013): 6.14 | Impact Factor (2014): 5.611}

the ratio of the lengths of the major and minor axes of this ellipse. Perfect circular polarization (only in theory) is obtainable if axial ratio is 1 .

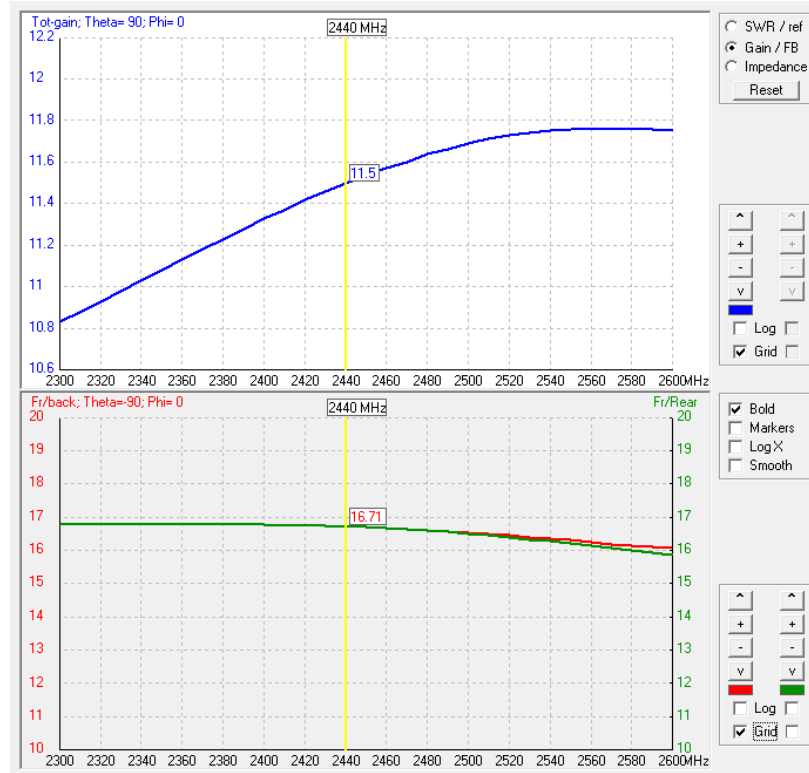

Figure 6: Helix antenna gain and FB ratio.

Using the 3D visualization on fig. 4 the student may readily observe the axial ratio of the studied antenna and see the pattern of the axial ratio, i.e. the axial ratio is not uniform in all directions in space.

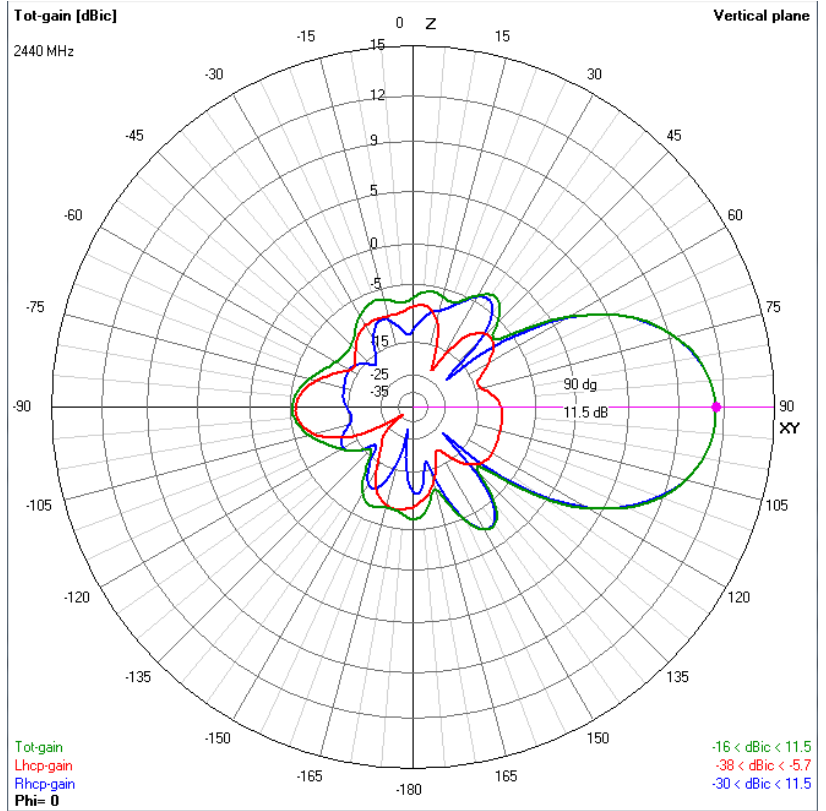

Figure 7: Helix antenna 2D gain diagram.

\section{Helix Antenna Construction}

The construction of the helix antenna is also very well observed using the $3 \mathrm{D}$ visualization (see fig. 1). The dimensions of the helix depend on the wavelength $\lambda$ of the radio waves that the antenna transmits or receives. The coil spacing is made approximately one-quarter of the wavelength $(\lambda / 4)$, while the coil diameter is approximately the wavelength divided by pi $(\lambda / \pi)$.

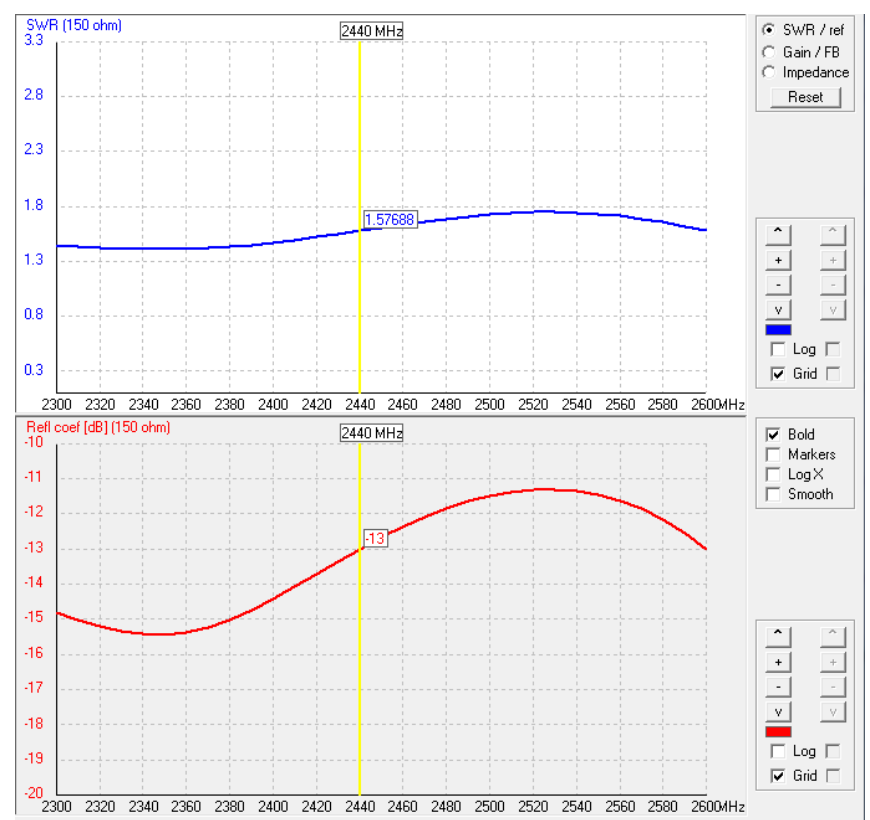

Figure 8: Helix antenna VSWR.

\section{Other Antenna Characteristics}

The helix antenna in axial mode is not a resonant antenna but a traveling wave antenna. Hence, it is a broadband antenna. The drawback of this characteristic is that the helix antenna is not tuned in resonance for the center frequency (2.4 GHz in this setup) and that's why its standing wave ratio (SWR) is not 1.0 .

Antenna impedance in axial mode is between 100 and 200 ohms (see fig. 9). If matched to $150 \mathrm{ohms}$ impedance the SWR may be observed on fig. 8. For the center frequency the SWR is 1.5 , but for the whole wide band from $2.3 \mathrm{GHz}$ to $2.6 \mathrm{GHz} \mathrm{SWR}$ never exceeds 1.8 , which is acceptable figure in most situations.

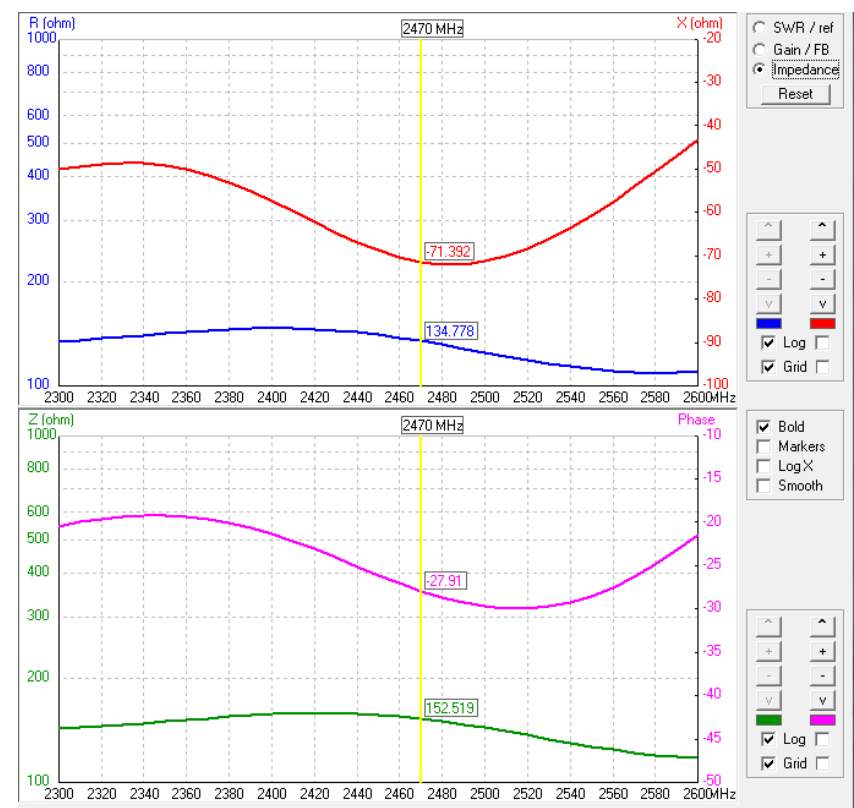

Figure 9: Helix antenna impedance diagram. 


\section{International Journal of Science and Research (IJSR) \\ ISSN (Online): 2319-7064}

Index Copernicus Value (2013): 6.14 | Impact Factor (2014): 5.611

\section{The Used Software}

The used software is 4 nec2, which is free software package for antenna analysis and 3D visualization, based on numerical integration method known as the Numerical Electromagnetics Code. The version incorporated in the current material is v5.8.15 and can be freely downloaded from the Internet [5-7].

The Numerical Electromagnetics Code, or NEC, is a numerical integration scheme for antenna simulation that was originally developed in FORTRAN programming language by Gerald Burke and Andrew Poggio of the Lawrence Livermore National Laboratory in the 1970s. Authors made the code public leading to its implementation for many platforms from mainframe computers to personal computers. NEC is commonly used software approach for designing antennas like television antennas, ham radio antennas, drone communication antennas, etc. NEC has limitations and for large antennas or microwave antennas other systems are commonly used.

\section{Conclusions}

Understanding radio-wave propagation and antenna physics is important benefit for students in physics and radioelectronics courses. 3D visualizations of antenna characteristics help not only master antenna design and theory, but also understand the hidden insights of radiophysics and electromagnetism. The presented approach delivers to students a valuable means of performing exercises in antenna designs and thus helps the process of traditional learning of physics and radio-electronics.

\section{References}

[1] Zabunov, S. \& Ivanov, K., 2003. Methods and Forms of Teaching "Information systems" and "Computer networks and communications" with the Use of the Internet. Annual of University of Mining and Geology "St. Ivan Rilski", Sofia, Bulgaria, 2003, Part IV: Humanitarian and Economic Sciences, Vol. 46, p. 141144

[2]Zabunov, S., 2004. A Language for Describing the Generating Structure of the Educational Material in the Individually Adaptive Learning Management System. International Conference on Computer Systems and Technologies - CompSysTech'2004, Rousse, Bulgaria, 17-18 June, 2004

[3] Zabunov, S. (2010). Rigid body motion in stereo 3D simulation. Eur. J. Phys., 31, 1345

[4] Zabunov, S., and Gaydarova, M., Rotating the flying disc in a stereo 3D simulation, Latin American Journal of Physics Education 7, 192 - 195 (2013).

[5] Adler, Dick (1993). "Information on the History and Availability of NEC-MOM Codes for PC's \& Unix". Applied Computational Electromagnetics Society Newsletter: 8-10.

[6] Burke, Gerald; Poggio, Andrew (1981). NEC Part III: User's Guide (PDF) (Technical report). Lawrence Livermore Laboratory.
[7] Lewallen, Roy (1991). "MININEC: The Other Edge of The Sword" (PDF). QST Magazine: 18-22.

\section{Author Profile}

Georgi Golemshinski is a researcher in the field of e-learning, geophysics and chemistry. He is interested in $3 \mathrm{D}$ visualization in the field of e-learning and 3D methods for analysis of geophysical imagery. Georgi Golemshinski leads active studies with regard to remote sensing using infrared thermal imaging. 